\section{Tabaquismo en el adulto mayor: un freno al envejecimiento saludable en la población chilena}

\author{
CRISTINA VIDAL ${ }^{\mathrm{a}}$, OSLANDO PADILLA ${ }^{1, \mathrm{~b}}$, \\ CLAUDIA BAMBS ${ }^{1,2}$
}

\section{Prevalence of smoking among Chilean older people}

\begin{abstract}
Background: Smoking is one of the main causes of death among adults worldwide. Aim: To characterize smoking among Chilean older people, according to sociodemographic and clinical variables. Material and Methods: Secondary analysis of data obtained during the National Health Survey 2009-10, selecting individuals aged 60 years and older. Expansion factors were used due to the complex design of the sample. Prevalence and characteristics of smoking were calculated, according to age, sex, educational level, marital status, healthcare insurance system and comorbidities. Results: Nineteen percent of older people were actual smokers, and $85 \%$ of these smokers were aged between 60 and 69 years. Forty-five percent were highly dependent to nicotine and $73 \%$ reported their intention to quit smoking. Conclusions: There is a high prevalence of tobacco smoking among Chilean older people. Prevention measures are needed.
\end{abstract}

(Rev Med Chile 2020; 148: 939-946)

Key words: Aged; Healthy Aging; Tobacco Smoking.
'Departamento de Salud Pública, Pontificia Universidad Católica de Chile. Santiago, Chile.

${ }^{2}$ Centro Avanzado de Enfermedades Crónicas. Pontificia Universidad Católica de Chile. Santiago, Chile. aprograma de especialidad médica en Pediatría, División de Pediatría, Dirección de Postgrado, Escuela de Medicina, Pontificia Universidad Católica de Chile. Santiago, Chile. bEstadístico.

Los autores declaran no tener conflictos de interés.

Financiamiento: Dra. Claudia Bambs recibe financiamiento del Centro Avanzado de Enfermedades Crónicas (ACCDiS, Fondap 15130011). ACCDiS no tuvo influencia en el diseño ni en la recolección, análisis o interpretación de los datos, ni en la preparación, revisión o aprobación del manuscrito.

Recibido el 24 de julio de 2019, aceptado el 27 de julio de 2020.

Correspondencia a:

Dra. Claudia Bambs

Departamento de Salud Pública UC, Edificio Académico, Av. Diagonal

Paraguay 362, piso 2, Santiago, Chile. cbambs@uc.cl
$\mathrm{E}$ $\mathrm{n}$ las últimas décadas se ha observado una acelerada transición epidemiológica y demográfica en Chile y Latinoamérica, con un acentuado envejecimiento poblacional ${ }^{1,2}$. Esto enfatiza la necesidad de promover un envejecimiento saludable, el cual se relaciona directamente con las conductas de salud de las personas a lo largo de la vida. Específicamente, no consumir tabaco es fundamental para un buen estado de salud en la tercera edad ${ }^{3}$.

El tabaquismo es uno de los factores más nocivos para la salud, repercutiendo sobre morbimortalidad, calidad de vida y costo económico para las personas y los países. Es la única sustancia comercializada legalmente que daña a todas las personas expuestas y mata a la mitad de sus consumidores crónicos. La epidemia del tabaquismo se ha ido trasladando hacia los países de bajos a medianos ingresos, los cuales concentran $80 \%$ del total de fumadores en el mundo, con la consecuente carga de morbimortalidad ${ }^{4,5}$.

Dentro de Latinoamérica, Chile ha liderado por décadas el ranking de consumo de tabaco ${ }^{6}$, alcanzando un máximo histórico de $40,6 \%$ en la población general de 15 años o más ${ }^{7}$. Si bien el consumo de tabaco en la población chilena ha bajado a $33,3 \%{ }^{8}$, el tabaquismo sigue siendo un importante problema de salud pública en nuestro país, responsable del $18,5 \%$ de las muertes anuales y 428.588 años de vida perdidos por discapacidad o muerte prematura ${ }^{9}$. Esto se traduce en un costo directo al sistema de salud chileno de más de un billón de pesos anuales, equivalente a $0,8 \%$ del producto interno bruto y a $11,5 \%$ del gasto anual en salud ${ }^{9}$. Por otra parte, el gasto promedio de un fumador en la compra de cigarrillos alcanza los \$ 311.000 anuales ${ }^{3}$, equivalente a aproximadamente un sueldo mínimo mensual en Chile, dinero que deja de 
ser invertido en bienes básicos como alimentación, vivienda, educación y la propia salud. Esto cobra especial relevancia en estratos sociales más bajos, los cuales, si bien presentan una menor prevalencia global de tabaquismo, exhiben un mayor consumo diario y mayor dependencia a la nicotina ${ }^{7,10}$.

Pese a la realidad del envejecimiento poblacional en Chile y a los múltiples efectos nocivos del consumo de tabaco sobre la salud de las personas, existe escasa información sobre el tabaquismo en el adulto mayor. El objetivo de este estudio fue caracterizar el tabaquismo en la población $\geq 60$ años, a partir de la Encuesta Nacional de Salud (ENS) de Chile 2009-10.

\section{Material y Métodos}

\section{Población y muestra}

Se realizó un análisis secundario de los datos de la población $\geq 60$ años obtenidos en la ENS 200910. Este estudio es representativo de la población general chilena de ambos sexos, de zonas urbanas y rurales, y se realizó a través de un muestreo probabilístico, estratificado y multietápico de 5.412 personas $\geq 15$ años. En la ENS 2009-10, los adultos mayores de 65 años o más fueron sobrerrepresentados en el muestreo (1.043 individuos), teniendo doble probabilidad de selección en el hogar, con el objetivo de obtener resultados de precisión estadística similar a los otros grupos de la población, aumentando la eficiencia de la muestra. La metodología de muestreo y el uso de factores de expansión en la ENS 2009-10 se encuentran publicados ${ }^{11}$.

El protocolo de la ENS 2009-10 fue aprobado por el Comité de Ética de Investigación de la Facultad de Medicina de la Pontificia Universidad Católica de Chile. Todos los participantes firmaron el consentimiento informado, incluida la autorización para el análisis diferido de sus resultados.

\section{Definición de variables}

En base a la definición del Servicio Nacional del Adulto Mayor de Chile (SENAMA), se consideró adulto mayor a todo individuo de $\geq 60$ años ${ }^{12}$. Los adultos mayores fueron clasificados en tres grupos etarios: 60-69 años, 70-84 años $y \geq 85$ años, y en tres estratos de nivel educacional (NEDU) según el total de años de escolaridad completa: bajo $(<8$ años), medio (8-12 años) y alto ( $\geq 12$ años), y según zona de residencia urbana o rural.
Se definió como fumadores a quienes reportaron haber fumado 100 o más cigarrillos durante su vida, y se categorizó la muestra total en tres grupos: no fumadores, exfumadores por 6 meses o más y fumadores actuales (diarios y ocasionales). Se clasificó como fumador diario a las personas que reportaron haber fumado al menos un cigarrillo al día en el último mes. Se excluyó del análisis a los exfumadores por menos de 6 meses (16 sujetos), por considerarse insuficiente para conducir análisis estadísticos.

La variable previsión de salud se clasificó en "sistema público FONASA", "ISAPRE u otra" (la cual incluyó la previsión de las Fuerzas Armadas y de Orden), y "ninguna". El estado civil se clasificó en "con pareja" (casados o convivientes), "viudo" y "otros" (solteros, separados, anulados o divorciados). El nivel de actividad física se midió mediante el cuestionario Global Physical Activity Questionnaire (GPAQ) ${ }^{13}$, agrupándose en "bajo", "moderado" y "alto". El estado nutricional se clasificó según el índice de masa corporal (IMC), en "enflaquecido" $\left(<18,5 \mathrm{~kg} / \mathrm{m}^{2}\right)$ ", "normal" $\left(18,5-24,9 \mathrm{~kg} / \mathrm{m}^{2}\right)$, “sobrepeso" $\left(25-29,9 \mathrm{~kg} / \mathrm{m}^{2}\right)$ y "obesidad" ( $\left.\geq 30 \mathrm{~kg} / \mathrm{m}^{2}\right)$ "11.

Se evaluó la presencia de comorbilidades en base al autorreporte de diagnóstico médico y cuestionarios sobre hipertensión arterial (HTA), diabetes mellitus (DM), colesterolemia total elevada (CT > $200 \mathrm{mg} / \mathrm{dl}$ ), enfermedad pulmonar obstructiva crónica (EPOC) y síntomas depresivos en el último año. Las mediciones de presión arterial, colesterolemia total y glicemia en ayunas se realizaron en el hogar de las personas, según protocolos estandarizados ${ }^{11}$. El deterioro cognitivo $y$ dependencia en la vida diaria se definieron con un puntaje $<13$ en el test de Minimental (MMSE) ${ }^{14}$ $y \geq 6$ en el test de Pfeffer ${ }^{15}$.

Se caracterizó el hábito tabáquico entre los adultos mayores fumadores diarios según el autorreporte del consumo promedio de cigarrillos al día en el último mes y según la intención de dejar de fumar y el grado de dependencia a la nicotina. Se clasificó como alta dependencia a la nicotina a quienes reportaron fumar su primer cigarrillo antes de una hora desde despertar ${ }^{11}$.

\section{Análisis estadístico}

Se calcularon medidas de tendencia central para variables continuas y proporciones para variables categóricas. Para el análisis estadístico 
se utilizaron los factores de expansión, considerando el carácter de muestreo complejo de la ENS 2009-10. Los datos se expresaron como frecuencia (\%) e intervalo de confianza (IC) 95\%, o media y desviación estándar (DE), según el tipo de variable. Las tablas muestran las prevalencias expandidas, representativas de la población chilena. Para la asociación de variables y ajuste por confundentes se realizaron regresiones logísticas múltiples y modelos de análisis de covarianza (ANCOVA).
Se calcularon Odds Ratio (OR) ajustados y sus IC 95\%. Los análisis se realizaron con el programa SPSS v. 17.0 (SPSS Inc., Chicago, USA), módulo de muestras complejas. Se definió un nivel de significancia estadística de $\mathrm{p}<0,05$.

\section{Resultados}

La Tabla 1 describe la muestra de adultos mayores según categorías de consumo de tabaco

Tabla 1. Caracterización del consumo de tabaco en adultos mayores chilenos, según variables sociodemográficas y clínicas. Chile, ENS 2009-10

\begin{tabular}{|c|c|c|c|c|c|}
\hline & $\begin{array}{c}\text { Total } \\
(\mathrm{n}=2.267 .420, \\
100 \%)\end{array}$ & $\begin{array}{c}\text { No } \\
\text { fumadores } \\
(n=1.184 .408 \\
52,2 \%)\end{array}$ & $\begin{array}{l}\text { Exfumadores por } \\
6 \text { meses o más } \\
\text { (n=648.723, } \\
28,6 \%)\end{array}$ & $\begin{array}{c}\text { Fumadores } \\
\text { actuales } \\
\text { (n }=434.288 \\
19,2 \%)\end{array}$ & Valor-p \\
\hline $\begin{array}{l}\text { Edad, \% (IC 95\%) } \\
60-69 \text { años } \\
70-84 \text { años } \\
85 \text { y más }\end{array}$ & $\begin{array}{c}60,2(55,6-64,6) \\
34,5(30,3-39,0) \\
5,3(3,7-7,6)\end{array}$ & $\begin{array}{c}49,7(43,6-55,8) \\
42,8(36,8-48,9) \\
7,5(4,9-11,5)\end{array}$ & $\begin{array}{c}62,5(53,4-70,7) \\
34,2(26,3-43,1) \\
3,3(1,6-6,5)\end{array}$ & $\begin{array}{c}85,3(78,1-90,4) \\
12,5(8,0-18,9) \\
2,3(0,6-8,3)\end{array}$ & 0,000 \\
\hline Sexo femenino, \% (IC 95\%) & $55,5(50,6-60,3)$ & $65,9(59,7-71,6)$ & $40(31,5-49,2)$ & $50,5(39,5-61,5)$ & 0,000 \\
\hline $\begin{array}{l}\text { Nivel educacional, media años de } \\
\text { estudios completos } \pm D E\end{array}$ & $7,71 \pm 0,26$ & $6,67 \pm 0,33$ & $8,63 \pm 0,50$ & $9,19 \pm 0,55$ & 0,000 \\
\hline $\begin{array}{l}\text { Previsión de salud, \% (IC 95\%) } \\
\text { FONASA } \\
\text { ISAPRE u otra (incluye FF. AA.) } \\
\text { Ninguna } \\
\text { Total }\end{array}$ & $\begin{array}{c}84,0(80,1-87,3) \\
11,7(9,0-15,1) \\
4,3(2,6-7,1) \\
100\end{array}$ & $\begin{array}{c}88,3(83,7-91,7) \\
6,9(84,6-10,1) \\
4,9(2,6-9,0) \\
100\end{array}$ & $\begin{array}{c}79,5(70,7-86,2) \\
15,2(9,9-22,7) \\
5,3(1,9-13,6) \\
100\end{array}$ & $\begin{array}{c}79,2(67,8-87,3) \\
19,5(11,5-31,0) \\
1,3(0,4-4,1) \\
100\end{array}$ & 0,011 \\
\hline Zona Urbana, \% (IC 95\%) & $83,2(79,4-86,5)$ & $80,3(74,9-84,7)$ & $85,1(75,9-91,1)$ & $88,6(81,9-93,0)$ & 0,169 \\
\hline $\begin{array}{l}\text { Estado civil, \% (IC 95\%) } \\
\text { Con pareja } \\
\text { Viudo } \\
\text { Otro }\end{array}$ & $\begin{array}{l}59,3(54,6-63,8) \\
22,5(19,3-26,2) \\
18,2(14,6-22,5)\end{array}$ & $\begin{array}{l}54,1(47,9-60,1) \\
25,9(21,5-30,8) \\
20,1(14,9-26,5)\end{array}$ & $\begin{array}{l}64,2(54,7-72,6) \\
19,3(13,6-26,6) \\
16,6(10,4-25,3)\end{array}$ & $\begin{array}{l}66,1(55,5-75,3) \\
18,4(11,2-28,7) \\
15,5(10,1-23,0)\end{array}$ & 0,232 \\
\hline $\begin{array}{l}\text { Nivel actividad física, \% (IC 95\%) } \\
\text { Bajo } \\
\text { Moderado } \\
\text { Alto }\end{array}$ & $\begin{array}{l}41,7(37,2-46,3) \\
21,4(17,5-25,9) \\
36,9(32,3-41,7)\end{array}$ & $\begin{array}{l}46,1(40,1-52,2) \\
21,9(16,9-27,8) \\
32,1(26,3-38,4)\end{array}$ & $\begin{array}{l}39,9(30,9-49,7) \\
24,5(16,3-35,2) \\
35,5(26,8-45,3)\end{array}$ & $\begin{array}{c}32,4(37,2-46,3) \\
15,6(10,0-23,4) \\
52(41,1-62,8)\end{array}$ & 0,037 \\
\hline $\begin{array}{l}\text { Estado nutricional, \% (IC 95\%) } \\
\left.\text { Normal (IMC } 18,5-24,9 \mathrm{~kg} / \mathrm{m}^{2}\right) \\
\text { Enflaquecido }\left(\text { IMC }<18,5 \mathrm{~kg} / \mathrm{m}^{2}\right) \\
\text { Sobrepeso }\left(\text { IMC } 25-29,9 \mathrm{~kg} / \mathrm{m}^{2}\right) \\
\text { Obeso }\left(\text { IMC } \geq 30 \mathrm{~kg} / \mathrm{m}^{2}\right)\end{array}$ & $\begin{array}{c}24,7(20,4-29,7) \\
1,1(0,5-2,5) \\
40,7(36,0-45,6) \\
33,4(29,1-38,1)\end{array}$ & $\begin{array}{c}23,6(18,7-29,3) \\
0,9(0,4-1,9) \\
38,5(32,6-44,7) \\
37,1(31,0-43,6)\end{array}$ & $\begin{array}{c}26,4(16,9-38,6) \\
0,6(0,1-2,3) \\
41,4(31,9-51,6) \\
31,7(24,0-40,5)\end{array}$ & $\begin{aligned} 25,3 & (16,3-37,0) \\
2,4 & (0,4-12,2) \\
45,6 & (34,4-57,2) \\
26,7 & (18,3-37,1)\end{aligned}$ & 0,550 \\
\hline Sospecha HTA, \% (IC 95\%) & $68,2(63,2-72,8)$ & $72,6(66,6-77,8)$ & $62,9(51,6-72,9)$ & $64,9(54,2-74,3)$ & 0,186 \\
\hline Sospecha DM, \% (IC 95\%) & $28,0(23,4-33,1)$ & $31,3(25,0-38,3)$ & $21,9(14,5-31,6)$ & $27,8(18,0-40,3)$ & 0,257 \\
\hline CT elevado, \% (IC 95\%) & $47,5(40,9-54,2)$ & $48,7(40,5-57,0)$ & $42,9(29,7-57,1)$ & $52,0(36,7-67,0)$ & 0,637 \\
\hline Antecedente de EPOC, \% (IC 95\%) & $7,7(5,6-10,6)$ & $4,9(3,2-7,2)$ & $11,5(6,3-20,0)$ & $9,9(5,2-17,9)$ & 0,048 \\
\hline Depresión, \% (IC 95\%) & $14,5(11,3-18,4)$ & $13,8(9,5-19,7)$ & $14,1(9,4-20,7)$ & $16,8(9,2-28,6)$ & 0,835 \\
\hline Deterioro cognitivo, \% (IC 95\%) & $4,5(2,7-7,5)$ & $4,0(2,6-6,2)$ & $7,6(2,9-18,4)$ & $1,2(0,3-4,5)$ & 0,070 \\
\hline
\end{tabular}

*Se excluyó de este análisis 14 sujetos sin información sobre previsión de salud. HTA: hipertensión arterial. DD: diabetes mellitus. CT: colesterolemia total. EPOC: enfermedad pulmonar obstructiva crónica. 
Tabla 2. Caracterización del hábito tabáquico en adultos mayores fumadores diarios, según categorías de edad. Chile, ENS 2009-10

\begin{tabular}{|lcccc|}
\hline & $\begin{array}{c}\text { Total } \\
\text { (n= 360.859, } \\
\text { (100\%) }\end{array}$ & $\begin{array}{c}\mathbf{6 0 - 6 9} \text { años } \\
\text { (n= 311.129, } \\
\mathbf{8 6 , 2 \% )}\end{array}$ & $\begin{array}{c}\mathbf{2} \text { (n) años } \\
\text { (n= 49.730, }\end{array}$ & Valor-p \\
\hline Número de cigarrillos fumados al día, media \pm DE & $11,14 \pm 1,11$ & $11,78 \pm 1,24$ & $6,92 \pm 1,15$ & 0,005 \\
\hline Alta dependencia, \% (IC 95\%) & $45,0(32,7-57,8)$ & $46,5(32,8-60,8)$ & $35,2(17,6-58,0)$ & 0,392 \\
\hline Intención de dejar de fumar, \% (IC 95\%) & $72,6(61,5-81,5)$ & $74,3(61,7-83,8)$ & $62,3(40,2-80,2)$ & 0,312 \\
\hline
\end{tabular}

*Alta dependencia a la nicotina: auto-reporte de fumar el primer cigarrillo del día antes de una hora desde despertar.

y variables sociodemográficas y clínicas. Del total de adultos mayores, 19,2\% correspondió a fumadores actuales y 28,6\% a exfumadores por 6 meses o más. Del total de hombres adultos mayores, $21,3 \%$ reportó consumir cigarrillos actualmente, en comparación con $17,4 \%$ de las mujeres (datos no mostrados).

Dentro de los fumadores actuales, $85,3 \%$ correspondió al subgrupo más joven (60-69 años). Fumadores actuales y exfumadores reportaron más años de estudios completos que los no fumadores ( 9,2 y 8,6 versus 6,7 años, respectivamente; $\mathrm{p}<0,001)$.

El 59,3\% de los adultos mayores reportó vivir con su cónyuge o pareja y $22,5 \%$ reportó ser viudo, sin diferencias significativas según categorías de consumo de tabaco. El 40,7\% y 33,4\% presentaron sobrepeso y obesidad, respectivamente. Comparados con los no fumadores, los adultos mayores fumadores actuales presentaron una mayor proporción de sujetos enflaquecidos y una menor proporción de sujetos obesos, pero la asociación entre estado nutricional y categorías de consumo de tabaco no alcanzó significación estadística en el presente análisis $(\mathrm{p}=0,55)$. Se observó un mayor autorreporte de diagnóstico de EPOC entre los fumadores actuales y exfumadores comparados con los no fumadores $(9,9 \%$ y $11,5 \%$ versus $4,9 \%$, respectivamente; $\mathrm{p}=0,048)$. No se encontraron diferencias significativas para otras comorbilidades según categorías de consumo de tabaco.

De los adultos mayores fumadores actuales, $83,1 \%$ correspondió a fumadores diarios, cuyo hábito tabáquico se caracteriza en la Tabla 2. Entre los fumadores diarios, el consumo promedio de cigarrillos fue mayor en el subgrupo de 60-69 años $(11,78 \pm 1,24$ versus $6,92 \pm 1,15$ cigarrillos/ día en los $\geq 70$ años; $p<0,05)$. El $45 \%$ de los fu- madores diarios presentó alta dependencia física a la nicotina y $72,6 \%$ manifestó intención de dejar de fumar. El análisis multivariable demostró que por cada año de vida del adulto mayor aumentó en $12 \%$ la intención de dejar de fumar, ajustado por sexo y NEDU (OR = 1,12; IC 95\% 1,04-1,20; $\mathrm{p}=0,001)$ (datos no mostrados).

El panel de Figuras 1A a la 1D presenta la caracterización del tabaquismo en los adultos mayores, según sexo y NEDU. Se observó una mayor prevalencia de tabaquismo actual entre los adultos mayores de NEDU alto $(27,3 \%$ versus $22,7 \%$ y $15,2 \%$ en NEDU medio y bajo, respectivamente). Las mujeres de NEDU alto presentaron la prevalencia más alta de tabaquismo actual $(34,7 \%)$; sin embargo, estas diferencias no alcanzaron significación estadística $(\mathrm{p}=0,085)$. Adicionalmente, se observó un mayor promedio de consumo de cigarrillos en los hombres de NEDU alto (15,6 cigarrillos/día versus 12,6 cigarrillos/día en hombres de NEDU bajo), sin alcanzar significación estadística $(\mathrm{p}=0,085)$. En concordancia con el mayor consumo de cigarrillos al día, se observó que los adultos mayores de NEDU alto presentaron los mayores índices de dependencia física a la nicotina (55\%) y la mayor proporción de individuos con intención de dejar de fumar $(86,8 \%)$, alcanzando $100 \%$ entre los hombres de NEDU alto. Sin embargo, estas diferencias no alcanzaron significación estadística.

\section{Discusión}

El presente estudio corresponde al primer reporte de la prevalencia y caracterización del tabaquismo en los adultos mayores chilenos. La prevalencia encontrada $(19,2 \%)$ es mayor que la 


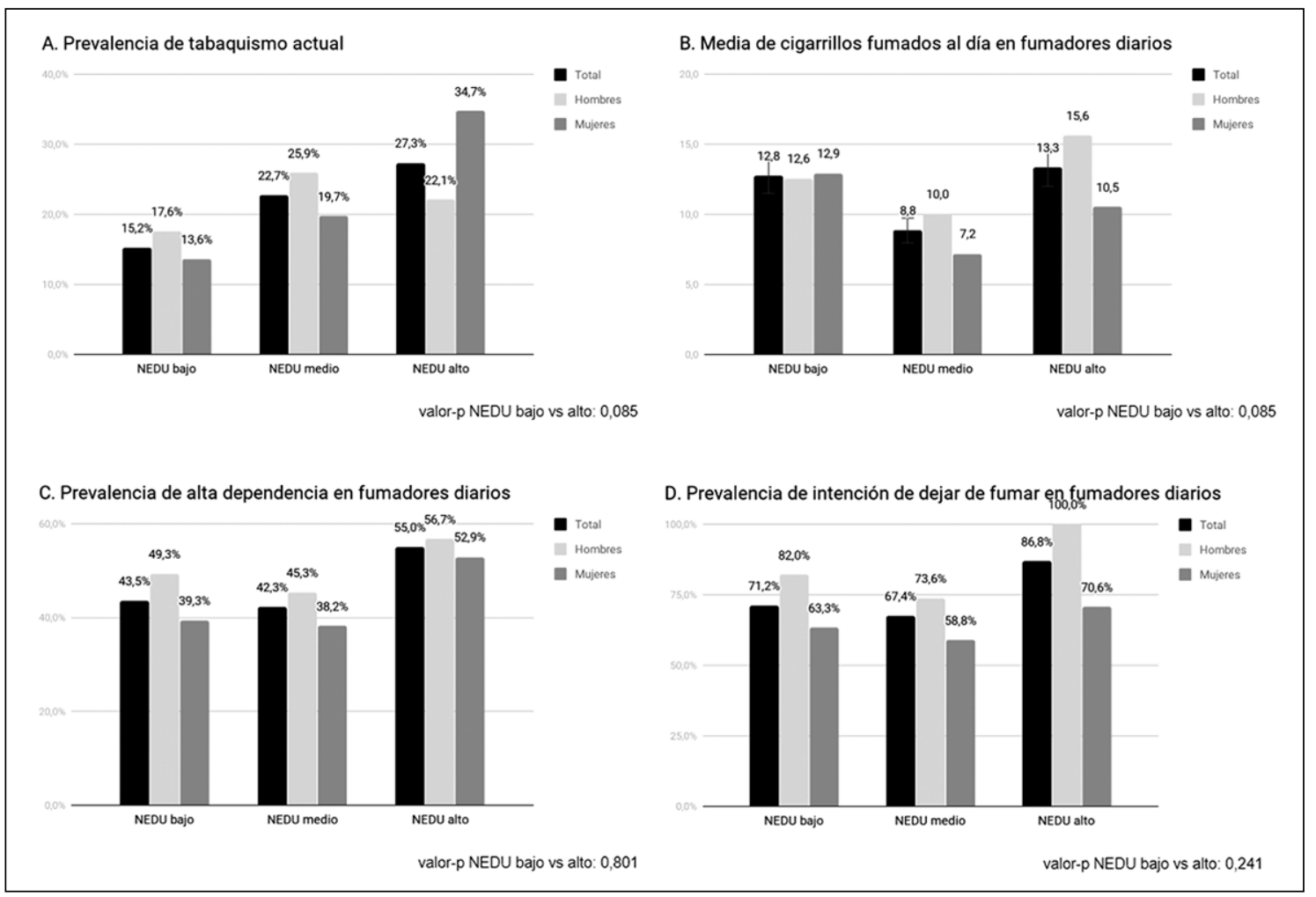

Figura 1. Panel de Figuras $1 \mathrm{~A}$ a la $1 \mathrm{D}$. Tabaquismo en adultos mayores chilenos según sexo y nivel educacional. Chile, ENS 2009-10. *NEDU bajo < 8 años de estudios completos, NEDU medio 8-12 años de estudios completos, NEDU alto > 12 años de estudios completos. NEDU: nivel educacional.

reportada en adultos mayores a nivel mundial $(13 \%)^{16}$. Estos hallazgos se encuentran en el contexto de la epidemia de tabaquismo que enfrenta nuestro país. Pese al avance en la implementación del Convenio Marco para el Control del Tabaquismo, en Chile aún un tercio de la población general fuma ${ }^{8}$, y las elevadas prevalencias de tabaquismo en mujeres y adolescentes indican que la epidemia está lejos de ser controlada ${ }^{17}$.

El hallazgo que la prevalencia de tabaquismo actual y el promedio de cigarrillos diarios fue mayor en el grupo más joven y en aquellos con mayor nivel educacional y pertenecientes al sistema privado de salud, puede explicarse, al menos en parte, por el mayor poder adquisitivo de estos grupos $^{10,18}$. Esto refuerza que el tabaquismo es sensible al precio de los cigarrillos ${ }^{19}$ y constituye evidencia adicional para apoyar el fortalecimiento de medidas de aumento de los impuestos y del precio de los cigarrillos para disminuir el consumo de tabaco entre los adultos mayores chilenos ${ }^{20}$. El hecho que la asociación entre nivel educacional y cantidad de cigarrillos diarios fumados no haya sido estadísticamente significativa se puede explicar por el bajo tamaño muestral del grupo de adultos mayores de NEDU alto $(\mathrm{n}=19)$.

Nuestros resultados en la población adulta mayor coinciden con lo reportado para población general según la ENS 2009-107 y estudios del Servicio Nacional para la Prevención y Rehabilitación del Consumo de Drogas y Alcohol (SENDA) ${ }^{21}$, en cuanto a que, si bien las personas de menor nivel educacional presentan una menor prevalencia de consumo de tabaco, concentran índices de dependencia a la nicotina. Por ende, los adultos mayores de menor nivel educacional podrían necesitar más apoyo para dejar de fumar y salir del círculo vicioso entre tabaquismo y pobreza ${ }^{10}$. Por lo anterior, nuestros hallazgos refuerzan la necesidad de fortalecer la red de apoyo a la cesación tabáquica a nivel del sistema público de salud en Chile. 
Destaca también la alta proporción de mujeres fumadoras de 60 años o más $(17,4 \%)$, respecto a lo descrito a nivel mundial $(8 \%)^{22}$. Si se considera que las mujeres chilenas son las más fumadoras de Latinoamérica ${ }^{6}$, y las adolescentes chilenas (entre 13 y 15 años) las más fumadoras el mundo $(39,2 \%)^{17}$, no es sorprendente que se mantenga esta tendencia entre la población de mujeres adultas mayores. Si bien el alto consumo de tabaco en la mujer chilena requiere un análisis que va más allá del alcance de este trabajo, cabe mencionar el contexto histórico, con fuertes corrientes de cambio político y social en el Chile de la década de 1960-69, que influyeron en cambios en la identidad y rol de las mujeres que actualmente están en edad adulta y adulta mayor ${ }^{23}$. A esto se agrega la influencia de fuertes estrategias de marketing dirigidas al público femenino ${ }^{24}$.

Los resultados de este estudio constituyen un insumo para fortalecer tanto las políticas públicas como el manejo clínico del adulto mayor fumador. La evidencia es clara en demostrar los beneficios de dejar de fumar, independiente de la edad en que se realice ${ }^{25}$. De hecho, la cesación tabáquica en la adultez mayor se asocia con una menor progresión de diversas comorbilidades y con mejoría de la calidad de vida ${ }^{26}$. Además, el adulto mayor podría destinar el ahorro económico derivado de dejar de fumar hacia otros bienes para mejorar su salud o calidad de vida en general. En cuanto al manejo clínico, nuestros resultados apoyan la recomendación de realizar la consejería breve de tabaco y contar con apoyo a la cesación tabáquica para los adultos mayores que deseen dejar de fumar, tal como se recomienda para la población general en la guía clínica chilena para el tratamiento del tabaquismo publicada el $2017^{27}$. Esto es relevante porque el apoyo a la cesación tabáquica sigue siendo el aspecto del convenio marco con menor nivel de implementación en Chile ${ }^{28}$. Cabe destacar que $55 \%$ de los adultos mayores chilenos no presenta alta dependencia física a la nicotina, de modo que el dejar de fumar podría ocurrir sin el imperativo de contar con apoyo farmacológico o psicosocial intensivo. Esto refuerza el rol de los profesionales de la salud en abordar este tema y motivar a los pacientes adultos mayores para dejar de fumar.

En cuanto a las limitaciones de nuestro estudio destaca que la población de adultos de 85 años o más fue considerablemente pequeña en nuestra muestra $(\mathrm{n}=86)$, por lo que no se pudo caracterizar adecuadamente. Además, al ser un estudio transversal no es posible establecer causalidad, sino solo asociaciones entre las variables estudiadas. Otra limitación es que, en concordancia con otros análisis publicados con datos de la ENS 2009-10, se utilizó el nivel educacional y la previsión de salud como aproximación al nivel socioeconómico, pudiendo corresponder a una evaluación incompleta de esta variable. Por otra parte, este estudio tiene las fortalezas de ser el primer estudio que caracteriza el tabaquismo en los adultos mayores chilenos y de contar con una muestra representativa de la realidad nacional, en la cual, prácticamente, no hubo datos perdidos para los análisis presentados, por lo que constituye un punto de partida para seguir estudiando este fenómeno en nuestro país.

En conclusión, los adultos mayores chilenos presentan una alta prevalencia de consumo de tabaco y esto atenta contra el envejecimiento saludable y funcional de nuestra población. Se requiere fortalecer el control del tabaquismo a nivel poblacional e incentivar el abordaje clínico del adulto mayor fumador por parte de los profesionales de la salud en nuestro país.

\section{Referencias}

1. Organización Mundial de la Salud (OMS). Informe mundial sobre el envejecimiento y la salud. 2015; Capítulo 3: Cambios demográficos y epidemiológicos; 45-53. Disponible en: http://www.who.int/ageing/publications/ world-report-2015/es/ [Consultado el 24 de septiembre de 2019].

2. Agar Corbinos L. Transición demográfica y envejecimiento en América Latina y el Caribe: hechos y reflexiones socio bioéticas. Acta Bioeth 2001; 7 (1): 27-41. Disponible en: https://scielo.conicyt.cl/scielo.php?scrip$\mathrm{t}=$ sci_arttext\&pid=S1726-569X2001000100003\&lng=es [Consultado el 24 de septiembre de 2019].

3. Bambs C, Alcantara J. Menos tabaco, más salud. En: Larraín F, Marín P, Valenzuela E. Editores. Cómo vivir bien 100 años. Santiago, Chile: Ediciones UC; 2016. p. 137-47.

4. World Health Organization (WHO), publicaciones. Tabaco. Disponible en: https://www.who.int/es/newsroom/fact-sheets/detail/tobacco [Consultado el 24 de septiembre de 2019].

5. Drope J, Schluger N, Cahn Z, Hamill S, Islami F, et al. The Tobacco Atlas. American Cancer Society and Vital Strategies. 6ta edición. Atlanta, EE. UU. 2018. p. 10, 25, 28. Disponible en: https://files.tobaccoatlas.org/wp-con- 
tent/uploads/2018/03/TobaccoAtlas_6thEdition_LoRes. pdf [Consultado el 24 de septiembre de 2019].

6. Organización Panamericana de la Salud (OPS). Informe sobre el control del tabaco en la Región de las Américas. Washington, D.C.: OPS; 2018. p. 7. Disponible en: http://iris.paho.org/xmlui/handle/123456789/49237 [Consultado el 24 de septiembre de 2019].

7. Ministerio de Salud (MINSAL) de Chile. Encuesta Nacional de Salud 2009-10. Tomo II. Capítulo V: resultados. Santiago, Chile: Ministerio de Salud; 2010. p. 153. Disponible en: https://www.minsal.cl/portal/url/item/ bcb03d7bc28b64dfe040010165012d23.pdf [Consultado el 24 de septiembre de 2019].

8. Ministerio de Salud (MINSAL) de Chile. Encuesta Nacional de Salud 2016-17. Santiago, Chile: Ministerio de Salud; 2017. Disponible en: https://www.minsal.cl/ wp-content/uploads/2017/11/ENS-2016-17_PRIMEROS-RESULTADOS.pdf [Consultado el 24 de septiembre de 2019].

9. Pichón-Riviere A, Bardach A, Caporale J, Alcaraz A, Augustovski F, Caccavo F, et al. Carga de Enfermedad atribuible al Tabaquismo en Chile. Documento Técnico IECS N ${ }^{\circ}$ 8. Instituto de Efectividad Clínica y Sanitaria, Buenos Aires, Argentina; 2014. p. 36, 40, 42, 44. Disponible en: http://www.eligenofumar.cl/wp-content/ uploads/2015/04/Informe-de-Carga-del-Tabaquismo-en-Chile.pdf [Consultado el 24 de septiembre de 2019].

10. Ciapponi A. Systematic review of the link between tobacco and poverty. World Health Organization (WHO); 2014. p. 26-31. Disponible en: http://apps.who.int/ iris/bitstream/10665/44453/1/9789241500548_eng.pdf [Consultado el 24 de septiembre de 2019].

11. Ministerio de Salud (MINSAL) de Chile. Encuesta Nacional de Salud 2009-10. Capítulo III: metodología. Chile: Ministerio de Salud, 2010. Disponible en: https:// www.minsal.cl/portal/url/item/bcb03d7bc28b64dfe040010165012d23.pdf [Consultado el 24 de septiembre de 2019].

12. Biblioteca del Congreso Nacional de Chile (BCN). Ley 19.828: Creación del Servicio Nacional del Adulto Mayor (SENAMA). Título I. 2002. Santiago, Chile. Disponible en: http://bcn.cl/1v31p [Consultado el 24 de septiembre de 2019].

13. Armstrong T, Bull F. Development of the World Health Organization Global Physical Activity Questionnaire (GPAQ). J Public Health 2006; 14: p. 66-70. Disponible en: https://www.ncbi.nlm.nih.gov/pubmed/20101923/ [Consultado el 24 de septiembre de 2019].

14. Folstein M, Folstein S, McHugh P. Mini-Mental State: a practical method for grading the cognitive state of patients for the clinician. J Psychiatr Res 1975; 12: p. 189-198. Disponible en: https://www.ncbi.nlm.nih.gov/ pubmed/1202204 [Consultado el 24 de septiembre de 2019].

15. Pfeffer R, Kurosaki T, Harrah C, Chance J, Filos S. Measurement of Functional Activities in Older Adults in the Community. J Gerontol 1982; 37: p. 323-9. Disponible en: https:/www.ncbi.nlm.nih.gov/pubmed/7069156 [Consultado el 24 de septiembre de 2019].

16. Marinho V, Laks J, Coutinho E, Blay S. Tobacco use among the elderly: a systematic review and meta-analysis. Cad Saúde Pública 2010; 26 (12): p. 2213-33. Disponible en: http://www.scielo.br/scielo.php?scrip$\mathrm{t}=$ sci_arttext\&pid=S0102-311X2010001200002\&lng=en [Consultado el 24 de septiembre de 2019].

17. Warren C, Jones N, Peruga A, Chauvin J, Baptiste J, Costa de Silva V, et al. Global Youth Tobacco Surveillance. Morbidity and mortality weekly report. Surveillance summaries. Washington, D.C. CDC 57(1): p. 1-28. 2008. Disponible en: https://www.researchgate. net/publication/5633013_Global_Youth_Tobacco_Surveillance [Consultado el 4 de febrero de 2019].

18. García A, Jiménez B. Envejecimiento y urbanización: implicaciones de dos procesos coincidentes. Investigaciones Geográficas. Instituto de Geografía, Universidad Autónoma de México (UNAM): México; 2016. 89: p. 58-73. Disponible en: https://www.sciencedirect.com/ science/article/pii/S018846111630022X [Consultado el 24 de septiembre de 2019].

19. Guindon G, Paraje G, Chaloupka F. The impact of Prices and Taxes on the Use of Tobacco Products in Latin America and the Caribbean. Systematic Review. Am J Public Health. (2015) 105: e9-e19. Disponible en: https://www.ncbi.nlm.nih.gov/pmc/articles/PMC4330839/ [Consultado el 24 de septiembre de 2019].

20. World Health Organization (WHO). Rising taxes on tobacco. Report on the Global Tobacco Epidemic. 2015. WHO. Disponible en: https://apps.who.int/iris/ bitstream/handle/10665/178574/9789240694606_eng. pdf?sequence $=1$ [Consultado el 24 de septiembre de 2019].

21. Servicio Nacional para la Prevención y Rehabilitación del Consumo de Drogas y Alcohol (SENDA). Ministerio del Interior y Seguridad Pública. Décimo segundo estudio nacional de drogas en población general de Chile, 2016. Observatorio chileno de drogas. p. 38. Diciembre, 2017. Disponible en: http://www.senda.gob. cl/wp-content/uploads/2017/12/InformeENPG2016.pdf [Consultado el 24 de septiembre de 2019].

22. Marie N, Freeman M, Fleming T, et al. Smoking Prevalence and Cigarette Consumption in 187 Countries, 
1980-2012. JAMA 2014; 311 (2): 183-92. Disponible en: https://jamanetwork.com/journals/jama/fullarticle/1812960. [Consultado el 24 de septiembre de 2019].

23. Stuven A, Cabello T, Crisóstomo B, Lozier M. La mujer ayer y hoy: un recorrido de incorporación social y política. Centro de Políticas Públicas UC, nº 61, julio 2013. ISSN 0718-9745. Disponible en: https://politicaspublicas.uc.cl/wp-content/uploads/2015/02/serie-no-61-lamujer-ayer-y-hoy-un-recorrido-de-incorporacion-social-y-politica.pdf [Consultado el 24 de septiembre de 2019].

24. Ravara S. Why it is important to address the gender and inequality gap on health. 4th International Network of Women Against Tobacco (INWAT) Europe Newsletter: marzo, 2017. Disponible en: https://www.tobaccofreehealthcare.org/static/uploads/files/inwat-4th-newsletter-wfxoqbytgkrg.pdf [Consultado el 24 de septiembre de 2019].

25. Becoña E. El valor de dejar de fumar cuando se es mayor. Rev Esp Geriatr Gerontol 2001; 36 Supl 1: 29-35. Disponible en: http://www.elsevier.es/en-revista-revis- ta-espanola-geriatria-gerontologia-124-articulo-el-valor-dejar-fumar-cuando-10021765 [Consultado el 24 de septiembre de 2019].

26. American Cancer Society. Benefits of Quitting Smoking Over Time. 2018. Disponible en: http://www.cancer. org/healthy/stay-away-from-tobacco/benefits-of-quitting-smoking-over-time.html [Consultado el 24 de septiembre de 2019].

27. Corvalán M, Véjar L, Bambs C, Pavié J, Zagolin M, Cerda J. Guías de Práctica Clínica para el Tratamiento del Tabaquismo. Santiago, Chile. Rev Chil Enferm Respir 2017; 33 (3): 167-75. Disponible en: https:// scielo.conicyt.cl/scielo.php?script $=$ sci_arttext\&pi$\mathrm{d}=$ S0717-73482017000300167\&lng=es [Consultado el 24 de septiembre de 2019].

28. Sandoval R, Blanco A, Martínez-López L, Caixeta R. Estado de la implementación del Convenio Marco para el Control del Tabaco en la región de las Américas. Salud pública Mex 2010; 52 (Suppl 2): p. 270-6. Disponible en: http://saludpublica.mx/index.php/spm/article/ view/8682/11124 [Consultado el 24 de septiembre de 2019]. 